\title{
Metodología para la transferencia de conocimiento de investigadores especialistas en logística a profesores de una universidad pública
}

\author{
Ileana Monsreal Barrera*, Jesús Escalante Euán** \\ Universidad Autónoma de Yucatán. Yucatán, México \\ Mario Monsreal Barrera*** \\ Centro Latinoamericano de Innovación en Logística, México \\ Recibido: 27 de mayo de 2015 / Aprobado: 25 de agosto de 2015
}

Resumen: Los empleos del futuro se caracterizarán cada vez más por la producción, intercambio y transformación de los conocimientos. Nuestras sociedades estarán plenamente inmersas en la asimilación de una oleada continua de nuevos conocimientos. La formación profesional tendrá que evolucionar forzosamente (Unesco, 2005). Este artículo describe la metodología y los resultados obtenidos mediante la implementación de un proyecto piloto que busca la transferencia de conocimiento de investigadores especialistas en logística de reconocimiento internacional y con conocimiento sobre las tendencias mundiales, hacia docentes de una universidad pública en Mérida, en el estado de Yucatán, México. La investigación se basó en la metodología de investigación-acción de McKernan.

Palabras clave: conocimiento / logística / competencias

\section{Methodology for Transferring Knowledge of Specialists Researchers in Logistics to Professors from a Public University}

AвsTRACT: The jobs of the future will be characterized by increasing production, interchange and transformation of knowledge. Our societies will be fully immersed in the assimilation of a continuous surge of new knowledge. The job training will be evolving necessarily (Unesco, 2005). This article introduces a pilot project that seeks the knowledge transfer of researchers specialized in logistics of international recognition and understanding of global trends of the subject matter, to teachers of a public University located in Mérida, Yucatán, Mexico. The research was based on the methodology of action research of McKernan consists of seven steps in him first cycle.

Key words: knowledge / logistic / competency

Correos electrónicos: *ileana.monsreal@correo.uady.mx, **jesus.escalante@upc. edu,***mmonsreal@cli-mexico.org 


\section{COMPETITIVIDAD Y LOGÍSTICA EN PERÚ Y MÉXICO}

Según el Foro Económico Mundial (Schwab, 2015), la competitividad es el conjunto de instituciones, políticas y factores que determinan el nivel de productividad. A su vez, este permite establecer el grado de prosperidad que puede ser ganado por una economía. En otras palabras, las economías más competitivas son capaces de producir altos niveles de ingreso para sus ciudadanos.

El mencionado organismo internacional genera un reporte global de competitividad, que es uno de los documentos de referencia más relevantes para comparar la situación de los países en relación con su desarrollo económico; esta medición implica el análisis de muy diversos factores que se han agrupado en doce pilares clave, reunidos a su vez en tres subíndices: requerimientos básicos (basic requirements), potenciadores de eficiencia (efficiency enhancers), innovación y factores de sofisticación (innovation and sophistication factors). Basándose en los resultados de estos tres grupos es que determinan la etapa de desarrollo en la que se encuentra un país, considerando tres etapas y sus transiciones, donde la tercera es la más avanzada.

Dentro de este reporte (Schwab, 2015), actualmente el Perú se ubica en el puesto 65 de un total de 144 países y figura en la etapa 2 , junto con otros 29 países; México se encuentra apenas un poco más arriba, en el lugar 61, en la transición de la etapa 2 a la 3 , junto con otros 23 países. En la etapa más avanzada (etapa 3 ) se encuentran 37 países. Para ser más explícitos en los resultados, en la tabla 1 se puede apreciar el nivel en el que se encuentran ambos países respecto a los doce pilares clave.

Entre los comentarios que se señalan en dicho documento en relación con el Perú, se mencionan la caída de cuatro posiciones y la preocupación por el funcionamiento de sus instituciones, junto con los avances insuficientes en la mejora de la calidad de su educación y la adopción tecnológica. El Perú necesita generar y utilizar el conocimiento para diversificar su economía hacia actividades más productivas, por lo que requerirá elevar la calidad de la educación que ahora no es capaz de proporcionar las habilidades necesarias para una economía cambiante.

Paralelamente, México, a pesar de las reformas estructurales recientes, cayó seis lugares, que se atribuyen principalmente al deterioro en la percepción del funcionamiento de las instituciones, la calidad de un sistema educativo que no parece cumplir con el conjunto de habili- 
dades que una economía mexicana cambiante requiere y su bajo nivel de implantación de las TIC, que es crucial para esta transformación.

Tabla 1

Doce pilares clave

\begin{tabular}{llcc}
\hline Subíndice & Pilares clave & Perú & México \\
\hline \multirow{3}{*}{ Requerimientos } & Instituciones & 118 & 102 \\
básicos & Infraestructura & 88 & 65 \\
& Estabilidad macroeconómica & 21 & 53 \\
& Salud y educación primaria & 94 & 71 \\
\hline \multirow{3}{*}{ Potenciadores } & Educación superior y capacitación & 83 & 87 \\
de eficiencia & Mercados de gran eficiencia & 53 & 86 \\
& Eficiencia en los mercados de trabajo & 51 & 121 \\
& Desarrollo del mercado financiero & 40 & 63 \\
& Preparación para la tecnología & 92 & 79 \\
Innovación y factores & Sofisticación de negocios & 43 & 10 \\
\cline { 2 - 3 } de sofisticación & Innovación & 72 & 58 \\
\hline
\end{tabular}

Elaboración propia

Es interesante observar en ambos casos los puntos de coincidencia. Primero, su ubicación en el ranking general, que los coloca en posiciones aproximadas; segundo, su paralelismo en cuanto al funcionamiento de sus instituciones y la necesidad de mejora en su calidad educativa para que cumplan con un conjunto de habilidades que requieren ambas economías.

La evolución de los mercados y el vertiginoso desarrollo de los negocios han convertido a la logística en una herramienta para alcanzar mayores índices de rentabilidad. Por eso, aprenderla se ha convertido en un desafío relevante, en una piedra angular de la cual dependen en gran medida los resultados de una empresa, donde hoy el nivel de eficiencia está más que nunca definido por la continuidad de la cadena en su conjunto (Woerner, 2015).

Así que, en los últimos años, la generación de conocimiento en logística y administración de la cadena de suministro ha tenido un notable crecimiento debido al interés en los beneficios económicos que se generan, derivados de una adecuada aplicación de este conocimiento en las organizaciones. 
La logística entendida como el manejo del flujo de materiales y productos dentro o entre organizaciones para transportar desde el punto de origen hasta el punto de destino dichos materiales o productos de manera eficiente y eficaz, tiene un impacto significativo no solo en la economía de la empresa sino en su capacidad de competir en los mercados que atiende. Esto, llevado a niveles macroeconómicos, influye en la competitividad de un país.

Lo anterior se ha podido distinguir claramente a partir del año 2007, a raíz de que el Banco Mundial publicó un estudio denominado Connecting to Compete: Trade Logistics in the Global Economy (Vincularse para competir: la logística del comercio internacional en la economía mundial), en el cual se señala que la logística del comercio internacional o la capacidad de conectarse con mercados internacionales para transportar bienes es crucial para que los países en desarrollo aumenten su competitividad, se beneficien de la globalización y logren reducir la pobreza en un mundo cada vez más integrado (Pecina, 2014).

El costo y la calidad de la logística tienen implicaciones fundamentales para el crecimiento económico sostenible. La mejora de un escalafón del desempeño logístico lleva en promedio a una ganancia en productividad laboral de cerca del $35 \%$. Esto es fundamental para América Latina y el Caribe (OECD, 2013).

El estudio mencionado plantea la medición del desempeño logístico de cada país considerando diversos indicadores y se han realizado mediciones en los años 2007, 2010, 2012 y 2014. En el caso específico de Perú y México, ocuparon las siguientes posiciones en los respectivos años, como puede observarse en la tabla 2 :

Tabla 2

Índice de desempeño logístico

\begin{tabular}{ccccc}
\hline & \multicolumn{2}{c}{ Perú } & \multicolumn{2}{c}{ México } \\
\cline { 2 - 5 } Año & $\begin{array}{c}\text { Índice de } \\
\text { desempeño logístico }\end{array}$ & $\begin{array}{c}\text { Ubicación } \\
\text { en el ranking }\end{array}$ & $\begin{array}{c}\text { Índice de } \\
\text { desempeño logístico }\end{array}$ & $\begin{array}{c}\text { Ubicación } \\
\text { en el ranking }\end{array}$ \\
\hline 2007 & 2,73 & 61 & 2,8 & 56 \\
2010 & 2,61 & 72 & 3,04 & 44 \\
2012 & 2,91 & 59 & 3,02 & 46 \\
2014 & 2,78 & 76 & 3,11 & 47 \\
\hline
\end{tabular}

Elaboración propia, con información obtenida del reporte global de competitividad del Foro Económico Mundial. 
A diferencia de la similitud en la ubicación en el ranking del reporte global de competitividad, Perú y México tienen marcada diferencia en los indicadores logísticos; Perú no ha podido superar su indicador de manera consistente, por lo que su ubicación en el ranking general ha sido oscilatorio; México, por su parte, ha dado pasos más firmes en ese sentido, manteniéndose por encima de 3 puntos y con una ubicación dentro del ranking que supera a la del Perú.

La vinculación entre logística y competitividad se refiere a la capacidad de capturar valor a partir de disminuir los costos y hacer más eficiente los procesos de abastecimiento, producción y comercialización de bienes. Esto reviste importancia tanto para las empresas como para el sector público (Canitrot, 2013).

Para el caso de América Latina, después de tener en cuenta el nivel de desarrollo de los países, gracias a las mejoras introducidas en los servicios logísticos, se podría potenciar la productividad laboral y reducir los costos comerciales que puedan estar asociados a una mayor sofisticación de los bienes exportados.

El desarrollo de estructuras profesionales y educativas en América Latina, que gestionen de manera integral y moderna los servicios logísticos, facilitaría la innovación de servicios de valor agregado a la carga.

Es necesario que los programas de educación se adapten más a las nuevas tecnologías y se promuevan programas interdisciplinarios que contemplen de forma holística la práctica logística y la gestión de cadenas de suministro, incluyendo, entre otras, prácticas de integración, sincronización, riesgos de la cadena y sostenibilidad.

Estos programas de aprendizaje y de formación continua deben permitir el desarrollo del capital humano al servicio de las competencias laborales, por lo que es fundamental el vínculo entre el sector empresarial y la academia (OECD, 2013).

Un marco integrado de la política de logística, una mejor gobernanza que promueva el transporte, el impulso de la educación logística, el buen uso de las TIC al servicio de la logística, la mejora en los procesos aduaneros, así como el fomento de la competencia, son algunos de los factores que pueden reducir los costos logísticos (OECD, 2013). 


\section{LA ENSEÑANZA DE LA LOGÍSTICA}

Hasta hace unos años, las principales opciones en el ámbito de educación logística se centraban en cursos y diplomados en comercio exterior, mientras que los aspectos logísticos solo eran una parte menor en carreras profesionales como ingeniería industrial, señala Cynthia Perisic (2012), gerente general de la Asociación Logística de Chile (ALOG): "Al tomar mayor importancia las tareas logísticas, el mercado obligó a las instituciones educativas a darle un espacio más relevante al tema dentro de su oferta, surgiendo carreras universitarias y de formación técnica, con especialización en el área logística".

La formación de los profesores es otro tema que concentra la atención de los especialistas de esta área. Ante la pregunta ¿quiénes están enseñando?, los expertos responden que se trata de profesionales provenientes de la empresa privada que se capacitan en docencia. Formar académicos integrales es, sin duda, otro desafío para las instituciones de educación y capacitación en logística, principalmente porque, como explica Jozsef Harmat (citado por Woerner, 2015), el tema de la formación en logística es tan amplio como ambicioso. Se trata casi de una gama infinita de disciplinas, que muchas veces nos hace caer en contradicciones vitales, porque por una parte queremos formar técnicos especialistas, a pesar de que la logística es conceptualmente generalista.

Según el reporte "Logística global 360" del Instituto Mexicano del Transporte, (citado por García, 2005) a principios del siglo XXI, en el Foro de Cooperación Económica de Asia-Pacífico (APEC), se creó un grupo de trabajo sobre transportación que investigó y analizó, entre otras, las consideraciones sobre aspectos de capacidad laboral especializada. Los estudios revelaron 32 habilidades laborales requeridas para diseñar planteamientos logísticos, agrupados en cuatro grandes categorías: habilidades fundamentales, analíticas, técnicas y de relación interpersonal, las cuales se desglosan en la tabla 3.

Ante esto, las habilidades de una persona formada en logística implican en sí un gran reto para el profesor, ya que requiere no solo del conocimiento teórico sino de una actualización constante sobre el conocimiento que se va gestando, y de la experiencia práctica sobre el uso del conocimiento para enfrentar los nuevos problemas que se generen en las diversas organizaciones. Es decir, que deberá entrar en un proceso continuo de aprendizaje autorregulado. 
Tabla 3

Habilidades laborales requeridas para diseñar planteamientos logísticos adecuados

\begin{tabular}{|c|c|}
\hline & Habilidades \\
\hline $\begin{array}{l}\text { Habilidades } \\
\text { fundamentales }\end{array}$ & $\begin{array}{l}\text { - Regulaciones y políticas gubernamentales } \\
\text { - Tecnologías disponibles para la logística y la transportación } \\
\text { - Entorno global de los negocios internacionales } \\
\text { - Entorno específico de nuestro negocio (mercado) } \\
\text { - Relaciones laborales internacionales } \\
\text { - Varias modalidades de transportación } \\
\text { - Interfaces de las varias modalidades de transportación } \\
\text { - Identificación y entendimiento de nuevas leyes }\end{array}$ \\
\hline Habilidades analíticas & $\begin{array}{l}\text { - Análisis (geográfico y humano) de los impactos del entorno } \\
\text { - Análisis económico y financiero } \\
\text { - Análisis de políticas públicas } \\
\text { - Planeación estratégica } \\
\text { - Habilidad para pronosticar } \\
\text { - Análisis de futuros } \\
\text { - Análisis de sistemas } \\
\text { - Análisis de la ética y la filosofía moral }\end{array}$ \\
\hline $\begin{array}{l}\text { Habilidades } \\
\text { técnicas personales }\end{array}$ & $\begin{array}{l}\text { - Aplicaciones computacionales en cadenas de logística y la } \\
\text { transportación intermodal } \\
\text { - Administración de la tecnología especializada } \\
\text { - Habilidades para la modelación } \\
\text { - Logística y procesos de la cadena de suministros y gestión } \\
\text { de la transportación internacional } \\
\text { - Compilación, análisis y manejo de la información y datos } \\
\text { - Habilidades de mercadeo } \\
\text { - Experiencia en transportación intermodal y logística } \\
\text { internacional }\end{array}$ \\
\hline $\begin{array}{l}\text { Habilidades de relación } \\
\text { interpersonal }\end{array}$ & $\begin{array}{l}\text { - Habilidades para el manejo administrativo general } \\
\text { - Habilidades para el servicio al cliente } \\
\text { - Habilidades para comunicarse con eficiencia } \\
\text { - Habilidades para saber escuchar } \\
\text { - Habilidades para las ventas } \\
\text { - Habilidades para escuchar y construir alianzas } \\
\text { - Habilidades para construir el equipo de trabajo } \\
\text { - Negociación y administración de conflictos } \\
\text { - Habilidades para dirigir y liderar }\end{array}$ \\
\hline
\end{tabular}

Fuente: "Logística global 360””, Instituto Mexicano del Transporte (García, 2005) 
De acuerdo con UPS (2013), una de las empresas logísticas más grandes en el ámbito mundial, la logística es el arte y la ciencia de hacer llegar las cosas exactamente donde y cuando tienen que estar. Este sentido eminentemente práctico de un área reciente y de tanta relevancia para la competitividad de las empresas de alcance global, vuelve un reto su enseñanza, por lo que es esencial que el docente de logística conozca cuáles son las competencias que se esperan de un estudiante formado en el área y se prepare al menos en aquellas que le corresponde trabajar con sus alumnos.

\section{SITUACIÓN EN LA UNIVERSIDAD AUTÓNOMA DE YUCATÁN (UADY)}

En el año 2004, la Facultad de Ingeniería Química inaugura la licenciatura en Ingeniería Industrial Logística, primera en su tipo en México y gran parte de Latinoamérica. Desde su inicio, uno de los primeros problemas que enfrentó la Facultad fue la búsqueda de profesores que dieran soporte al proceso de enseñanza, dado que el conocimiento generado hasta ese momento en el área de Logística provenía principalmente de las grandes empresas transnacionales y, por tanto, no se contaba con profesores que cumplieran el perfil de docentes formados en empresas de estas características.

$\mathrm{Al}$ inicio de este proyecto (enero de 2013), la Facultad contaba con un profesor cuyas competencias en el área de logística las obtuvo directamente de una empresa, y otro profesor que había llevado un doctorado específico en la referida área. Los demás docentes que daban soporte a la licenciatura tenían formación en ingeniería industrial principalmente y otras ramas de la ingeniería, por lo que la velocidad de cambio en el conocimiento de la logística y la necesidad de mejorar las competencias de los profesores de la licenciatura llevó a la interrogante: ¿se puede implementar algún programa que permita a los profesores de la Facultad de Ingeniería Química de la UADY, que imparten materias relacionadas con la logística, recibir a la vez acceso al conocimiento del área y a técnicas docentes apropiadas para su transmisión de con el nuevo enfoque por competencias?

\section{DISEÑO DE LA INVESTIGACIÓN}

De acuerdo con lo anterior, se estaba ante una problemática de corte microsocial focalizada en la acción social, ya que no pretendía expli- 
car una tendencia general de la sociedad, sino estudiar un caso muy particular de una comunidad académica que busca elevar la calidad educativa en la logística mediante un proceso innovador a través de una prueba piloto, que podría ser perfeccionada y reaplicada posteriormente, a otra escala y en distinta comunidad.

$\mathrm{Al}$ hacer referencia a la idea de Kleining (1982), dada la naturaleza del proyecto, se estaba ante un primer nivel de abstracción y todos los métodos de investigación tienen el mismo origen en las técnicas cotidianas, pero a partir de ellas los métodos cualitativos son el primer nivel de abstracción, y los métodos cuantitativos el segundo (Flick, 2007). Así que el proyecto se apegó a una metodología de investigación cualitativa, y se entiende por tal "cualquier tipo de investigación que produce hallazgos a los que no se llega por medio de procedimientos estadísticos $u$ otros medios de cuantificación” (Strauss y Corbin, 2002, p. 8).

Existen diversos métodos para abordar la investigación cualitativa, dadas las características de este proyecto se aplicó el método de la investigación-acción. La finalidad de este tipo de investigación es resolver problemas cotidianos e inmediatos (Álvarez-Gayou, 2003) y mejorar prácticas concretas. Su propósito fundamental se centra en aportar información que guíe la toma de decisiones para programas, procesos y reformas estructurales.

Como menciona Sandín (citado por Sampieri, Collado y Lucio, 2008), entre las principales características de los estudios de esta naturaleza se encuentran las siguientes:

- La investigación-acción envuelve la transformación y mejora de una realidad (social, educativa, administrativa, etc.); de hecho, se construye desde esta.

- Parte de problemas prácticos y vinculados con un ambiente o entorno.

- Implica la total colaboración de los participantes en la detección de necesidades (ellos conocen mejor que nadie la problemática por resolver, la estructura que se va a modificar, el proceso que hay que mejorar y las prácticas que requieren transformación) y en la implementación de los resultados del estudio (Sampieri et al., 2008).

- McKernan (citado por Álvarez-Gayou, 2003) propone el proceso temporal de la investigación-acción, que consiste de varios ciclos subsecuentes. 
El primer ciclo de acción consiste en los intentos por definir claramente la situación o el problema. Posteriormente se pasa a la evaluación de las necesidades, estableciéndose las limitaciones externas e internas del progreso. Esta revisión del problema debe propiciar que surjan ideas, propuestas o hipótesis, las cuales no se asumen como soluciones. Luego se realiza un plan general de acción, que se lleva a la práctica y se evalúa. En esta evaluación, los participantes buscan comprender los efectos y lo que han aprendido. En el segundo ciclo, o en los sucesivos, se produce una nueva definición revisada del problema para realizar otra evaluación de las necesidades, a partir de las cuales surgen más ideas o hipótesis que llevan a la revisión del plan. Este se vuelve a poner en práctica para realizar nuevamente una evaluación, con lo cual se vuelven a tomar decisiones, incluyendo la comprensión y la explicación obtenidas. El ciclo puede reiniciarse tantas veces como sea necesario (Álvarez-Gayou, 2003).

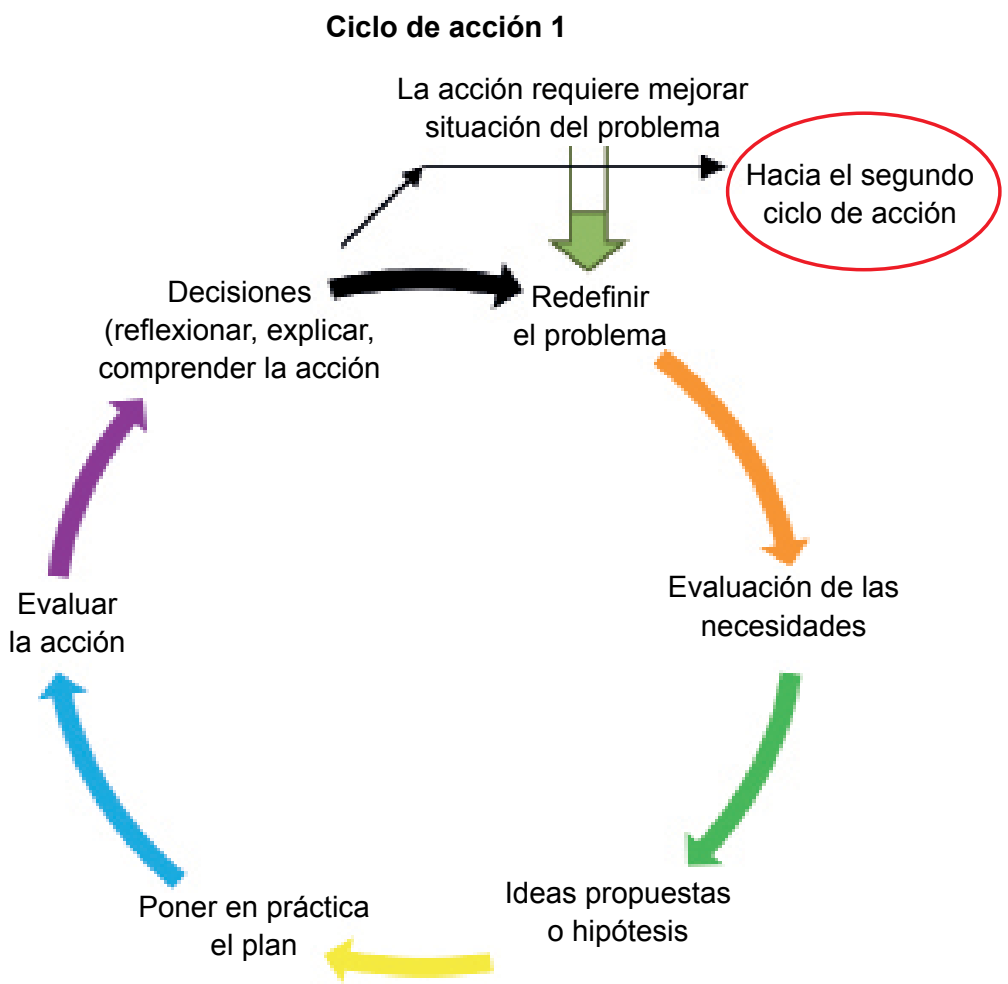

Desarrollar el plan de acción

Figura 1. Proceso temporal de la investigación según McKernan (2001)

Fuente: Álvarez-Gayou (2003) 
Este modelo de McKernan es el que se implementó al proyecto, dentro de un primer ciclo, lo que implicó, en términos generales:

- definir el problema

- identificar las necesidades

- establecer una propuesta para subsanar el problema

- desarrollar la propuesta

- evaluar la propuesta

- revisar la propuesta contra los resultados obtenidos buscando comprender los efectos

- sugerir acciones para un segundo ciclo

\section{TÉCNICAS DE RECOLECCIÓN DE DATOS}

Dada la naturaleza del problema del presente proyecto, se registró información de los sujetos de estudio (profesores) y el mecanismo de transformación (certificado). Las fuentes de información fueron los propios sujetos y el oferente del mecanismo de transformación el Centro Latinoamericano de Innovación en Logística-México.

El tiempo del que se dispuso estuvo sujeto a dos demandas: la del órgano que financió el proyecto, cuyo plazo fue de 12 meses, y los tiempos solicitados por la propia universidad, en cuyo caso fueron cinco meses para la impartición del certificado y los restantes para completar la investigación.

De acuerdo con lo anterior, se utilizaron las siguientes técnicas:

- aplicación de encuestas

- observación participante no estructurada, y

- entrevistas semiestructuradas y centradas en el problema

El cuestionario es propiamente un instrumento de la investigación cuantitativa, pero dada la premura del tiempo para la impartición del certificado, sirvió como acercamiento a la entrevista individual estructurada más propia de los métodos cualitativos.

Según McCraken (citado por Sandoval, 1996), el cuestionario cumple varias funciones dentro de la entrevista individual estructurada, dos de ellas se aplicaron al presente caso: 1) asegurar que el investigador cubra todo el terreno, en el mismo orden, para cada entrevistado, y 2) mantener la distancia con el entrevistado. 


\section{ESTRATEGIA DE MUESTREO}

Para profundizar en la investigación se seleccionó una muestra de los participantes con los que se tuvieron entrevistas semiestructuradas. Sandoval (1996) señala que en el muestreo teórico se acude a una lógica comprensiva que depende de los avances que se van alcanzando en el entendimiento del problema estudiado, durante el progreso mismo de la investigación. En relación a la selección de los participantes se orienta por dos principios: pertinencia y adecuación. El primero tiene que ver con la identificación y logro del concurso de los participantes que pueden aportar la mayor y mejor formación a la investigación, de acuerdo con los requerimientos teóricos de esta última; la adecuación se refiere a contar con datos suficientes disponibles para desarrollar una completa y rica descripción del fenómeno, preferiblemente cuando se ha alcanzado la etapa de la saturación.

La selección de la muestra se apegó a los requerimientos de un muestreo teórico abordado de manera gradual, de acuerdo a las fases descritas por Sandoval (1996).

La primera etapa fue la aplicación del cuestionario a todos los participantes, que permitiera recabar información general pero relevante de estos, a fin de obtener una valoracion inicial; posteriormente se definieron los criterios apropiados que respetaran los principios de pertinencia y adecuación para una exploración más profunda a una parte de los participantes. Estos criterios fueron los siguientes:

1) Ser participante del certificado.

2) Ser profesor de la UADY.

3) Impartir o haber impartido clases en la licenciatura de Ingeniería Industrial Logística o en la Maestría en Administración de Operaciones que cuenta con una orientación en logística.

4) Participar en algún cuerpo académico o grupo disciplinar reconocido por la Facultad y haber realizado investigación preferentemente relacionada con el área de logística.

5) Haber participado activamente durante las sesiones de clase.

6) Desear colaborar con el proyecto.

Ya con estos elementos, durante las sesiones se observó la participación e interés de los profesores participantes en el curso y se seleccionó a los que los cumplieron, por lo que se obtuvo una muestra de un tercio de los participantes. 


\section{DESARROLLO DEL PROYECTO}

El proyecto se inició con un acercamiento al Centro Latinoamericano de Innovación en Logística-México (CLI-México), para solicitar su asesoría sobre las alternativas para mejorar las competencias de los docentes del área de logística. Cabe comentar que el CLI-México es un centro de investigación especializado en logística que inició operaciones en México en 2010, como resultado del esfuerzo conjunto del Zaragoza Logistic Center y del Logyca (Colombia), ambos participantes de la red SCALE del Instituto Tecnológico de Massachusetts.

De acuerdo con el programa marco de trabajo y la revisión sistemática de los objetivos, competencias y criterios, y como respuesta a la solicitud de asesoría, el CLI-México sugirió el diseño de un programa que fuera ad hoc a las necesidades de la institución, para lo cual hizo una propuesta inicial; ante esto, y para precisar las necesidades de mejora en las competencias docentes requeridas y orientarlas al modelo educativo para la formación integral que estaba incorporándose a los programas de la Universidad Autónoma de Yucatán, se realizó una revisión bibliográfica sobre antecedentes del modelo de competencias y sus repercusiones en el rol del profesor, así como las competencias requeridas a un profesional del área de logística.

Con esta información, se tuvieron reuniones con el CLI-México con el fin de suministrar la información sobre las necesidades específicas del modelo por competencias en cuanto a estrategias didácticas y mecanismos de transferencia del conocimiento para el desarrollo de competencias de logística en los niveles de licenciatura y posgrado, y se fueron definiendo los temas que serían propios para tratar durante esta prueba piloto en la que se conformaría cada módulo del certificado, así como la modalidad y tiempo que se estimaba llevaría, que quedaron de la siguiente manera:

Temática: - Módulo I. Introducción a la logística y cadena de suministro

- Módulo II. Métodos de enseñanza para la cadena de suministro

- Módulo III. Competencias logísticas en contextos emergentes

Modalidad: Mixta

Duración: 120 horas 
Una vez definido lo anterior, el CLI-México ubicó a los expertos de los respectivos temas dentro de la red, trabajó con ellos los detalles y conformó la propuesta del certificado.

Para iniciar el desarrollo de la prueba piloto del programa diseñado, se trabajó con el CLI-México la programación de fechas de las sesiones para adecuarla a la disponibilidad de los instructores y a las necesidades de la institución.

Asimismo, se solicitó a la Dirección de la Facultad que designara a los profesores que consideraba pertinentes para formar parte de esta prueba. También invitó a otros profesores para que participen en el programa y adicionalmente invitó a otras instituciones de la entidad por si consideraban que algunos de sus docentes requerían de este programa.

Como resultado de las acciones anteriores, se inscribieron 12 profesores, 10 de esta institución y 2 de una institución del puerto de Progreso.

Paralelamente, se realizó una revisión bibliográfica para extraer un glosario básico de conceptos de logística como material preliminar del certificado, para el caso de que hubiera profesores que no tuvieran noción alguna del área.

Posteriormente se preparó un cuestionario que permitiera obtener información general pero relevante de los participantes y se sometió a revisión por parte del CLI-México. A su vez, y basándose en el glosario mencionado, se preparó una evaluación de inicio que permitiera medir la homogeneidad o no del grupo y el conocimiento, por parte de los participantes, de algunos conceptos básicos del área.

$\mathrm{Al}$ inicio del certificado, y de acuerdo con la metodología, la primera etapa consistió en un diagnóstico inicial de los participantes, se aplicó el cuestionario y evaluación elaborados con anterioridad; posteriormente, el CLI-México calificó la evaluación preliminar y la comentó con los instructores, durante la plática de inicio para darles una perspectiva general de las características de la institución, de sus programas y del grupo.

Con esta información también se hizo un mapeo de los participantes, como paso previo a la selección de la muestra para un segundo acercamiento, de acuerdo con los criterios señalados en la metodología.

Durante el transcurso del programa se monitoreó a través de la observación a los participantes e instructores. Se mantuvieron pláticas de retroalimentación mutua con los instructores, las cuales permitieron ir 
ajustando el programa a las condiciones del grupo. A su vez, hubo espacios de diálogo entre instructores y participantes para acordar mejores mecanismos de transferencia del conocimiento, y se observó la participación e interés de los asistentes a los cursos. La retroalimentación de los instructores en ese sentido permitió completar el criterio cinco para la selección de la muestra, como describe Sandoval (1996) en su metodología de muestreo teórico, lo que llevó a seleccionar a un tercio de ellos, cuatro profesores, para un segundo acercamiento.

Así también, se realizaron evaluaciones generales sobre el desempeño del instructor al final de cada módulo, que fueron compartidas posteriormente con los instructores.

Al finalizar el programa, la evaluación de los participantes se efectuó de acuerdo con los criterios definidos por el CLI-México en su propuesta, entre los que se encuentra la realización de un producto académico, respecto del cual se hicieron requerimientos específicos a los participantes.

Finalmente, y para efectos de profundizar en la percepción de los participantes del programa en relación con esta prueba piloto, se realizó el segundo acercamiento a los profesores seleccionados como muestra. Para ello se llevaron a cabo sesiones individuales de entrevistas semiestructuradas, en las fechas que fueron factibles para los profesores.

\section{RESULTADOS}

La asistencia promedio de los profesores fue del $93 \%$, y como resultado del proceso de evaluación aplicado y reportado por el CLI-México obtuvieron las calificaciones que se muestran en la tabla 4 .

Estos resultados se comparan posteriormente en la figura 2 con los obtenidos en la prueba inicial, lo que permite ver la particularidad de cada caso.

En relación con los instructores, se utilizó una escala de evaluación de 1 al 5, donde 5 es la mayor calificación, para evaluarlos en relación a dos aspectos principales: su desempeño y el contenido de sus respectivos módulos (tabla 5); los participantes del curso otorgaron las siguientes calificaciones a cada uno de ellos. 
Tabla 4

Evaluación de participantes

\begin{tabular}{cccccc}
\hline \multicolumn{5}{c}{ Evaluación de asistentes al Diplomado en Enseñanza de la Logística } \\
\hline Participante & $\begin{array}{c}\text { Calificación } \\
\text { Módulo I }\end{array}$ & $\begin{array}{c}\text { Calificación } \\
\text { Módulo II }\end{array}$ & $\begin{array}{c}\text { Calificación } \\
\text { Módulo III }\end{array}$ & $\begin{array}{c}\text { Calificación } \\
\text { por trabajo } \\
\text { final }\end{array}$ & $\begin{array}{c}\text { Calificación final, } \\
\text { promedio de módulos } \\
\text { y trabajo final }\end{array}$ \\
\hline $\mathbf{1}$ & 100 & 100 & 90,91 & 85,71 & 94,15 \\
$\mathbf{2}$ & 90,91 & 100 & 90,91 & 100 & 95,45 \\
$\mathbf{3}$ & 72,73 & 81,82 & 90,91 & 85,71 & 82,79 \\
$\mathbf{4}$ & 100 & 100 & 72,73 & 100 & 93,18 \\
$\mathbf{5}$ & 100 & 100 & 100 & 71,43 & 92,86 \\
$\mathbf{6}$ & 90,91 & 90,91 & 90,91 & 100 & 93,18 \\
$\mathbf{7}$ & 100 & 100 & 54,55 & 100 & 88,64 \\
$\mathbf{8}$ & 100 & 100 & 100 & 100 & 100 \\
$\mathbf{9}$ & 100 & 100 & 100 & 85,71 & 96,43 \\
$\mathbf{1 0}$ & 100 & 100 & 100 & 100 & 100 \\
$\mathbf{1 1}$ & 100 & 90,91 & 100 & 85,71 & 94,16 \\
$\mathbf{1 2}$ & 81,82 & 90,91 & 72,73 & 85,71 & 82,79 \\
\hline
\end{tabular}

Elaboración propia

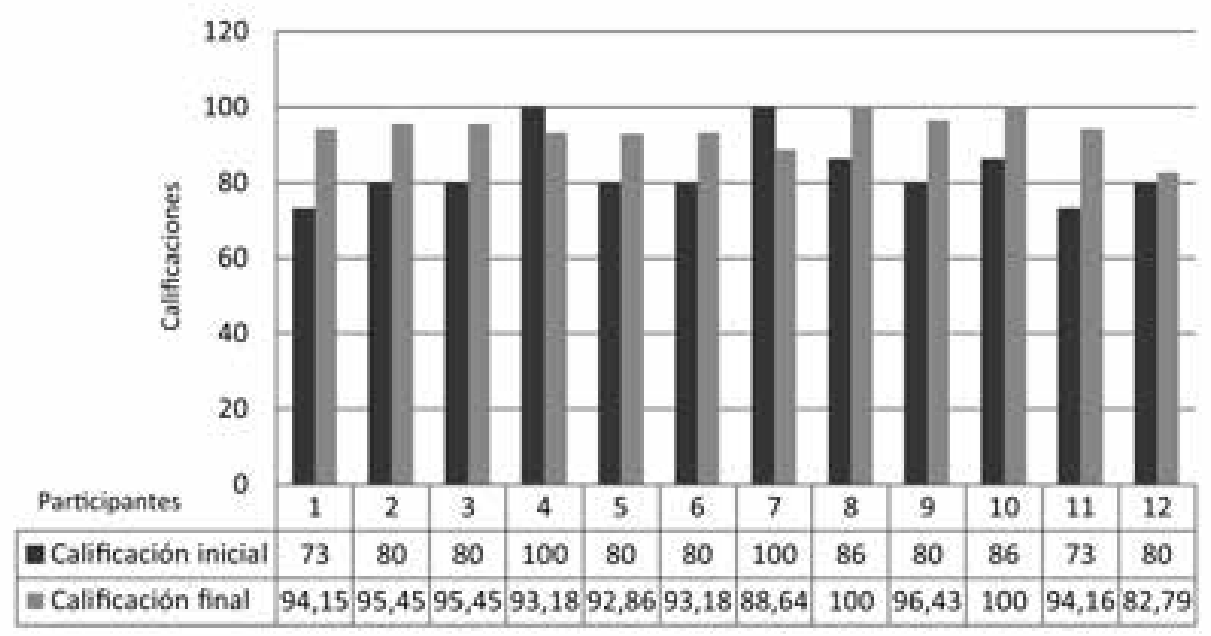

Figura 2. Calificación inicial y final del diplomado Elaboración propia 
Tabla 5

Evaluación de instructores

\section{Evaluación de instructores y contenido del programa}

\begin{tabular}{|c|c|c|c|c|c|}
\hline $\begin{array}{l}\text { I. Desempeño del } \\
\text { instructor }\end{array}$ & $\begin{array}{c}\text { Instructor } \\
\text { A }\end{array}$ & $\begin{array}{c}\text { Instructor } \\
\text { B }\end{array}$ & $\begin{array}{l}\text { Instructor } \\
\text { C }\end{array}$ & $\begin{array}{l}\text { Instructor } \\
\text { D }\end{array}$ & $\begin{array}{l}\text { Promedio } \\
\text { general }\end{array}$ \\
\hline $\begin{array}{l}\text { 1. Tiene conocimiento y } \\
\text { experiencia en el tema }\end{array}$ & 5 & 4,5 & 4,7 & 4,9 & 4,8 \\
\hline $\begin{array}{l}\text { 2. Mantuvo claridad } \\
\text { en la presentación y } \\
\text { comunicación }\end{array}$ & 4,9 & 4,5 & 5 & 4,2 & 4,7 \\
\hline $\begin{array}{l}\text { 3. Contestó las preguntas } \\
\text { y aclaró dudas de } \\
\text { manera adecuada }\end{array}$ & 4,9 & 4,7 & 4,5 & 4,3 & 4,6 \\
\hline $\begin{array}{l}\text { 4. Manejó ejercicios, } \\
\text { dinámicas o casos }\end{array}$ & 5 & 48 & 4,6 & 4,7 & 4,8 \\
\hline $\begin{array}{l}\text { 5. Fue hábil para resumir } \\
\text { puntos básicos }\end{array}$ & 4,7 & 4,5 & 4,4 & 4,7 & 4,6 \\
\hline $\begin{array}{l}\text { 6. Manejó adecuadamente } \\
\text { el tiempo }\end{array}$ & 4,8 & 4,1 & 4,3 & 4,5 & 4,4 \\
\hline $\begin{array}{l}\text { 7. Usó equipo y material } \\
\text { didáctico }\end{array}$ & 5 & 4,8 & 4,7 & 4,6 & 4,8 \\
\hline $\begin{array}{l}\text { 8. Fue puntual en las } \\
\text { sesiones }\end{array}$ & 4,9 & 4,9 & 5 & 4,7 & 4,9 \\
\hline 9. Fomentó la participación & 4,9 & 4,3 & 5 & 4,9 & 4,8 \\
\hline $\begin{array}{l}\text { 10. Dirigió al grupo para } \\
\text { mantener discusiones } \\
\text { constructivas }\end{array}$ & 4,9 & 4,4 & 4,8 & 4,7 & 4,7 \\
\hline II. Contenido del módulo & & & & & $\begin{array}{l}\text { Promedio } \\
\text { general }\end{array}$ \\
\hline $\begin{array}{l}\text { 1. Se cubrió el objetivo } \\
\text { propuesto }\end{array}$ & 4,8 & 4,7 & 4,6 & 4,5 & 4,7 \\
\hline $\begin{array}{l}\text { 2. Aplicabilidad del } \\
\text { contenido }\end{array}$ & 4,8 & 4,7 & 4,4 & 4,4 & 4,6 \\
\hline 3. Estructura de la clase & 4,7 & 4,6 & 4,5 & 4,4 & 4,6 \\
\hline
\end{tabular}

Elaboración propia 
Respecto de las observaciones no estructuradas y comentarios realizados por participantes e instructores durante el programa, se pudo apreciar lo siguiente:

- De los participantes

- Algunos participantes comentaron que hubiera sido deseable la entrega de material al inicio de la certificación, pero la limitante planteada por los instructores radicó en el costo de los derechos de los casos utilizados, que debían ser cubiertos por cada participante y que se subsanó con el uso puntual por parte del instructor al momento de trabajarlos.

- No todos los participantes llegaban puntualmente a las sesiones, y dado que se encontraban en su área de trabajo algunos se ausentaban un momento para atender otros asuntos.

- Una limitante observada y también manifestada por los participantes fue el tiempo, que resultó insuficiente para tratar los temas.

- De los instructores

- Los cuatro instructores demostraron el dominio de sus respectivas especialidades en la materia y su experiencia en el manejo de grupos, cada uno con sus particularidades, que fueron interesantes de observar por parte de los participantes.

- Además, cada instructor procedía de diferentes países y su desarrollo profesional se había dado también en otras culturas, lo que enriqueció la clase por las disímiles problemáticas planteadas y resueltas desde su óptica intercultural.

- Del programa

- El programa propuesto por el CLI-México no pudo ser cubierto en su totalidad y los instructores tuvieron que hacer ajustes sobre la marcha del programa; según se observó, y por comentarios de los instructores, esto se debió en parte a que gran cantidad de la información utilizada, como los casos, debía ser trabajada en inglés, y los profesores participantes no tenían el nivel de lecturacomprensión del mencionado idioma. Además, la concentración de clases en módulos semanales de días completos limitaba la posibilidad de trabajar horas adicionales fuera de aula.

- El último módulo del programa contempló dos sesiones a distancia, las cuales fueron bien vistas y agradaron a la mayoría de los participantes.

Los principales resultados de las entrevistas semiestructuradas aplicadas a un tercio de los participantes fueron los siguientes: 
- Los cuatro profesores consideraron que obtuvieron nuevos elementos que les permitirán mejorar sus competencias docentes; dos de ellos fueron muy enfáticos al respecto. Los aspectos que más llamaron su atención fueron la experiencia vivencial con los instructores y la metodología para el manejo de casos (su uso, dirección de discusiones, la novedad de los casos presentados y la extrapolación a las realidades regionales que captaron el interés de los participantes).

- Tres profesores consideraron que hubo elementos novedosos, relacionados con las técnicas docentes, particularmente en el manejo de casos y el uso de juegos. El cuarto profesor declaró no ver algo novedoso pero señaló en una de las respuestas que llamó su atención la forma como algunos profesores abordaron ciertos temas y cómo dirigían las discusiones.

\section{CONCLUSIONES}

- En relación a la pregunta de investigación: ¿se puede implementar algún programa que permita a los profesores de la Facultad de Ingeniería Química de la UADY, que imparten materias relacionadas con la logística, tener acceso al conocimiento del área y a la vez a técnicas docentes apropiadas para la transmisión de estos, de acuerdo con el enfoque por competencias? La conclusión, con base en los resultados (véase la figura 2), es que a pesar de las limitaciones expresadas, el programa les permitió actualizar sus conocimientos y sus competencias docentes, obteniendo evaluaciones positivas en 10 de los 12 casos. Así también, cabe mencionar que los profesores entrevistados declararon que de la certificación en enseñanza de la logística obtuvieron nuevos elementos que les permitirá mejorar sus competencias docentes.

- El diseño de nuevas licenciaturas multidisciplinares y enfocadas al modelo por competencias demanda académicos con alta preparación teórica y práctica en sus áreas de enseñanza, por lo que este tipo de programas apoyados en expertos internacionales facilitan el acceso a la actualización de los docentes. Sin embargo, y dadas las características observadas en este grupo, solo cuatro profesores declararon tener experiencia y solo uno de ellos mencionó las empresas donde laboró, lo que dificultará educar en competencias del área a aquellos profesores que son evidentemente teóricos, por lo que en un segundo ciclo de investigación habría que valorar la posibilidad de comple- 
tarlos con estancias prácticas para los profesores, en este caso, en empresas donde se desarrollen actividades logísticas.

- Para un segundo ciclo habría que considerar las sugerencias que manifestaron los profesores entrevistados, entre las que cabe destacar una capacitación para la realización de proyectos de investigación del área, en la que se aborden problemáticas regionales, lo que sería de gran apoyo a los docentes universitarios para adquirir o mejorar otras competencias que les permitieran generar conocimiento en la región donde se desenvuelven.

- La asesoría del CLI-México como centro de investigación especialista en el área y su relación directa con los participantes de la red SCALE del Tecnológico de Massachusetts, fue fundamental para cumplir con el objetivo del proyecto, pues cuentan con investigadores reconocidos en todo el mundo, que continuamente están generando conocimiento actualizado del tema, docentes que imparten en programas específicos del área, y proyectos de investigación relacionados con empresas de diversas nacionalidades, incluyendo proyectos en Latinoamérica, lo que dio un panorama muy amplio para estructurar un programa según las necesidades de la UADY-FIQ y seleccionar instructores de alto nivel pero acordes con nuestra cultura y nuestros problemas.

- Transmitir a sus respectivos alumnos, en el corto plazo, los conocimientos de vanguardia adquiridos por el personal docente de cualquier institución, de manera especial en áreas eminentemente aplicadas, como es el caso de la logística, debe proporcionar una ventaja competitiva a los alumnos de dichas instituciones, la cual no fue posible medir en este proyecto dado su alcance. Sin embargo, sería deseable un proyecto que contemplara esta hipótesis. Lo que implicaría una investigación más extensa, profunda y de mayor duración.

\section{REFERENCIAS}

Álvarez-Gayou, J. L. (2003). Cómo hacer investigación cualitativa. México: Paidós Mexicana.

Canitrot, L. (2013). La logística como herramienta para la competitividad. Recuperado de http://www.camarco.org.ar/File/GetPublicFile?id=1061 
Fernández, S., y Trejo, A. (2013). Knowledge transfer, the case of a Mexican region. Global Conference on Business \& Finance Proceedings, 8(2), 1398-1407.

Flick, U. (2007). Introducción a la investigación cualitativa (2. ${ }^{a}$ ed.). Madrid: Ediciones Morata S.L.

García, R. J. (2005). Logística global $360^{\circ}$ : una nueva visión con el vínculo educativo y laboral. Publicacion Técnica (279).

Gil-Albarova, A., Martínez, A., Tunnicliffe, A., y Miguel, J. (2013). University students and the quality of the tutorial project. Evaluation and improvements [Estudiantes universitarios y calidad del plan de acción tutorial. Valoraciones y mejoras]. Revista de Docencia Universitaria, 11(2), 63-87.

Gonzales, V. (2002). El profesor universitario: ¿un facilitador o un orientador en la educación de valores? Pedagogía Universitaria, 7(4), 44-51.

Hernández, R., Fernández, C., y Baptista, P. (2008). Metodología de la investigación (4. ${ }^{\mathrm{a}}$ ed.). Vol. 1. México, D. F.: McGraw-Hill.

Krüger, K. (2006). El concepto de «sociedad del conocimiento». Revista Bibliográfica de Geografía y Ciencias Sociales, 11(683). Recuperado de http://www.ub.edu/geocrit/b3w-683.htm

López, A., y Farfán, P. (2010). El enfoque por competencias en la educación. Recuperado de http://www.congresoretosyexpectativas.udg. $\mathrm{mx} /$ Congreso\%205/Mesa\%203/ponencia6.pdf

Morales, G. (2009). El claroscuro de la formación por competencias. Xipe Totek: Revista Trimestral del Departamento Filosofía y Humanidades ITESO, 18(71), 193-215.

Moreno, T. (2012). La evaluación de competencias en educación. Revista Electrónica Sinéctica, 39, 1-21.

OECD (2013). Políticas para impulsar la logística en América Latina. Perspectivas Económicas de América Latina 2014: Logística y competitividad para el desarrollo. Doi: 10.1787/leo-2014-es

Pecina, M. (2014). Retos en la formación de profesionales logísticos. Negocios Globales. Logística, Transporte y Distribución. Recuperado de http://www.eumed.net/libros-gratis/2014/1372/ index.htm 
Perisic, C. (2012). El desafío de armonizar la teoría con las crecientes demandas laborales. Negocios Globales. Logística, Transporte y Distribución. Recuperado de http://www.emb.cl/negociosglobales/articulo.mvc?xid=1316

Sampieri, R., Collado, C. F., y Lucio, P. B. (2008). Metodología de la investigación. (4. ${ }^{a}$ ed.). Vol. 1. México, D. F.: McGraw-Hill.

Sandoval, C. A. (1996). Investigación cualitativa. Bogotá: Instituto Colombiano para el Fomento de la Educación Superior (ICFES).

Schwab, K. (2015). The Global Competitiveness Report 2014-2015. World Economic Forum. Recuperado de http://www.weforum. org/reports/global-competitiveness-report-2014-2015

Strauss, A., y Corbin, J. (2002). Bases de la investigación cualitativa: técnicas y procedimientos para desarrollar la teoría fundamentada. Medellín, Colombia: Universidad de Antioquia.

Unesco. (2005). Hacia las sociedades del conocimiento. París: Unesco.

UPS. (2013). Guía 15. Formas de aprovechar el poder de la logística: hechos, consejos y puntos de vista para tu negocio. La nueva logística UPS. Recuperado de http://lanuevalogistica.ups.com/ guia/UPS-Ways-Guide-PDF-MX.pdf

Woerner, A. (2015). Educación en logística. El aprendizaje y control de la cadena. Negocios Globales, Logística, Transporte y Distribución. Recuperado de http://www.emb.cl/negociosglobales/articulo.mvc ?xid=1171\&edi=57\&xit=educacion-en-logistica-el-aprendizaje-ycontrol-de-la-cadena 